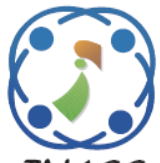

\title{
Blind Digital Images Tampering Detection Based on Singular Value Decomposition
}

\author{
Nidhal K. El Abbadi ${ }^{1} \quad$ Alyaa Mohsin ${ }^{2 *}$ \\ ${ }^{I}$ Department of Computer Science, Education College, University of Kufa, Najaf, Iraq \\ ${ }^{2}$ Department of Computer Science, Faculty of Computer Science and Mathematics, University of Kufa, Najaf, Iraq \\ * Corresponding author's Email: aalyam.zayi@uokufa.edu.iq
}

\begin{abstract}
The growing use of digital images in a wide range of applications, and growing the availability of many editing photo software, cause to emerge a challenge to discover the images tampering. In this paper, we proposed a method to detect the most important type of forgery image (copy and move). We suggested many steps to classify the image as forgery or non-forgery image, started with preprocessing (included, convert image to gray image, de-noising, and image resize). Then, the image will be divided into several overlapping blocks. For each block, feature extracted (used it as a matching feature) by using the singular value decomposition (SVD) transformation. According to these features, the pixels were collected in many main groups, and then these groups clustered to many subgroups. The weight for each main group can be determined by comparing the subgroups with each other according to suggested conditions. The number of subgroups and weights are used to classify images to forgery or non-forgery images. The accuracy of detection and classified the forgery images were up to $97 \%$. The suggested method is robust for tampered object rotation, scaling, and change of illumination.
\end{abstract}

Keywords: Computer vision, Copy-move, Image forgery, SVD, Tampered object.

\section{Introduction}

Nowadays, images are very important in communication media. There is believed that images can express about the accident or situation in a way that is clearer, and truth than words [1]. In the current technological world, Digital images play a very significant role in different huge fields such as the law court, military, medical records, news, scientific publication, and media. With advancements in digital images technology such as camera equipment, programs, and computer systems and the widespread use of digital images through the internet media, so, the digital images can be used as an important information point at the moment, due to the advanced technology and access to inexpensive forge devices of hardware and software. Therefore, it is easy to forge digital images without any observable elements. The deceiving of digital image forgery in many cases intentionally damages the recipient's knowledge. In such a case, digital image forgery detection has especially a crucial role in forensic to give authenticate to the image [2,3].

A forgery is a criminal work that supplies misleading information around a product or service. It is the operation of making, conform, or fake documents or objects with the purpose to deceive. Digital forgery is the operation of tampering documents or pictures for financial, social, or political gain [4].

There are three kinds of image forgery: retouching forgery, image splicing forgery, and image copy-move forgery [5].

The first kind of forgery, retouching forgery is moderate and less dangerous than other types of forgery. It does not modify or change image significantly, but Image retouching does improve or reduce the quality of the image properties. The second kind of forgery, splicing forgery is a method that contains a composition of multiple images combined to create the forgery. The third kind of forgery, Copy-move forgery is the same as splicing 
forgery. but in this type copied a portion of an image and pasted to the desired location of the same image. Copy-move forging can be divided into several types: simple Copy-move, Copy-move with a different rotation, and Copy-move with different scaling [2]. In this paper, we try to detect copy-move image forgery in addition to solve the problems of region scaling, rotation, and variation of illumination based on the Singular Value Decomposition (SVD).

Many papers used SVD, but in the current work, we use it differently. The diagonal matrix used for evaluation instead of using the three matrices results from applying SVD transformation.

In this way, for each block we convert the diagonal matrix values to one value (norm value) which represents the matching value between two blocks, this value does not affect by block geometric distortion such as scaling and rotation.

This method solving the problems of forgery detection, scaling, rotation, and illumination variation in one simple algorithm, where the other methods need to suggest an algorithm for each problem.

The rest of this paper is organized as follows: Section 2 illustrates the details of the literature survey. section 3 explained the suggested method (tools and algorithm), while section 4 introduces the results and discussion. finally, the conclusion and future work of the proposed algorithm are provided in section 5.

\section{Related works}

Bi and Pun (2018) [6] suggested to apply and improve a coherence-sensitive hazing process to determine the identical attribute in the image. Next, suggested a local bidirectional coherence fault to refine the identical attribute through the improved coherence sensitive seeking by iteration. The iterative operation will stop and show that identical attributes are stable if the difference in the host image's local bidirectional coherence fault is not greater than a given threshold. Finally, the local bidirectional coherence fault of each attribute is used to detect the region's copy-move forgery from a stable identical attribute. Detection precision was 96\%. However, for ideal conditions, other methods are performing better under common distortion, geometric distortions (e.g., rotation and scaling), and it is not tested for change of image illumination.

Pun and Chung (2018) [7] suggested a method to detect image forgery of the copy-move type includes two-stage localization. In the first phase, rough localization is used Simple Linear Iterative Clustering (SLIC) to divide the picture into meaningful regions (superpixels). Thereafter, the
Weber Local Descriptor (WLD) is suggested to compute and elicit the feature from each region. Then, a threshold used to extract the corresponding regions. Finally, Euclidean distance is used to remove the weak features of the regions and get the rough suspected identify. In the second phase, precise localization includes circular blocks with various radii are slip over high rough expected regions to elicit the block features by applying the Discrete Analytic Fourier-Mellin Transform (DAFMT). Detection precision was $97 \%$. However, the detection efficiency of this method can be improved by using a scheme that involves constructing a scaling invariant rather than detecting multiple host images using different circular blocks (CBs) radii. This method is not tested for change of image illumination.

Texture and region features combine to detect forging images introduced by Isaac and Wilscy (2018) [8]. The texture feature Rotation Invariant Cooccurrences between adjacent LBP (RICoLBP) is implemented in the regions. The region is captured by using the region based methods such as waveletbased region discovery, saliency-based region discovery, and edge-based region discovery. Support Vector Machine (SVM) and positive matrix factorization are used to optimize the extracted features and classification them. Detection precision was $97 \%$. However, post-processing operations such as noise addition, scaling, rotation, change of image illumination, etc. have not been covered in this technique for efficient forgery detection.

Kashyap, Agarwal, and Gupta (2018) [9] proposed an approach for using both Singular Value Decomposition (SVD) and Cuckoo Search (CS) algorithms to detect copy-move forgery. Uses the Cuckoo Search algorithm is to create custom parameter values for various tampered images that are used in the block-based system for copy-move forgery detection. Detection precision was $94 \%$.

Although CMFD-CS applies to most of the copymove forged images but observed that the method did not test for rotation and change of image illumination. In the proposed algorithm, the SVD used in different ways than in the paper introduced in [9], algorithm CMFD-CS has calculated the inverse of the natural logarithm of each singular value and the outcomes are summed for each sub-blocks, then the Euclidean distance is checked, summing of values will produce redundant values (not unique values), and this will highly affect on the matching blocks or detection. CMFD-CS can't find reliably matched blocks in uniform texture regions or when the duplicate regions are too small.

While the current proposed algorithm focus on the finding norm of singular value matrix which is a 
unique value, and this value used for determining block matching. It works very well for uniform texture regions whatever it's the size, and works well when the region scaled or rotates, unlike the CMFDCS.

The current algorithm processed all these drawbacks by using the SVD as a matching algorithm. The suggested method enhances the image forgery detection even when (scaling with different sizes (shrink or enlarge) and rotate with any angle). In addition to detecting image forgery when a change of image illumination.

\section{Proposed method}

In the last decades, a new mathematical algorithm emerged that change a way of thinking. This method starts in small fields and expands rapidly to many other fields. One of the basic and most important tools of modem numerical analysis, particularly numerical linear algebra [10]. This technique is the singular value decomposition (SVD), it is utilized to extract geometric and algebraic features of the image. Because SVD features described as stability, scaling, and rotation invariance, SVD has been popularly applied to discover copy-move forging. SVD decomposes a matrix into many matrices, displaying some of the beneficial and important properties of the original matrix [11], it is a method to factorization matrix into three matrices. For any matrix $\left(A \in R^{m \times n}\right)$ there are three decomposed matrices.

$$
A=U S V^{T}
$$

Where

- $\mathrm{U}$ is an $\mathrm{m} \times \mathrm{m}$ orthogonal matrix (called the left singular vectors).

- $\quad \mathrm{S}$ is an $\mathrm{m} \times \mathrm{n}$ diagonal matrix.

- $\mathrm{V}$ is an $\mathrm{n} \times \mathrm{n}$ orthogonal matrix (called the right singular vectors).

We assume at this work $m \geq n$. In the case of $m=n$, there are only non-zero positive values in the diagonal of the diagonal matrix. The values of the diagonal matrix (singular value (S)) are in descending order. i.e., $S_{1} \geq S_{2} \geq \ldots \geq S_{n} \geq 0$. the diagonal matrix values are square roots of the eigenvalues of matrix $\mathrm{AA}^{\mathrm{T}}$ and the matrix $\mathrm{A}^{\mathrm{T}} \mathrm{A}$ [12]. Eq. (1) can be rewritten as:

$$
a_{i j}=\sum_{k=1}^{n} u_{i k} s_{k} v_{j k}
$$

SVD is a significant and major technique in numerous various applications such as [10]: object detection, signal processing, image compression, face recognition, watermarking, and noise removal.

\subsection{Methodology}

The suggested method for the detection of copymove image forgery is summarized in algorithm 1.

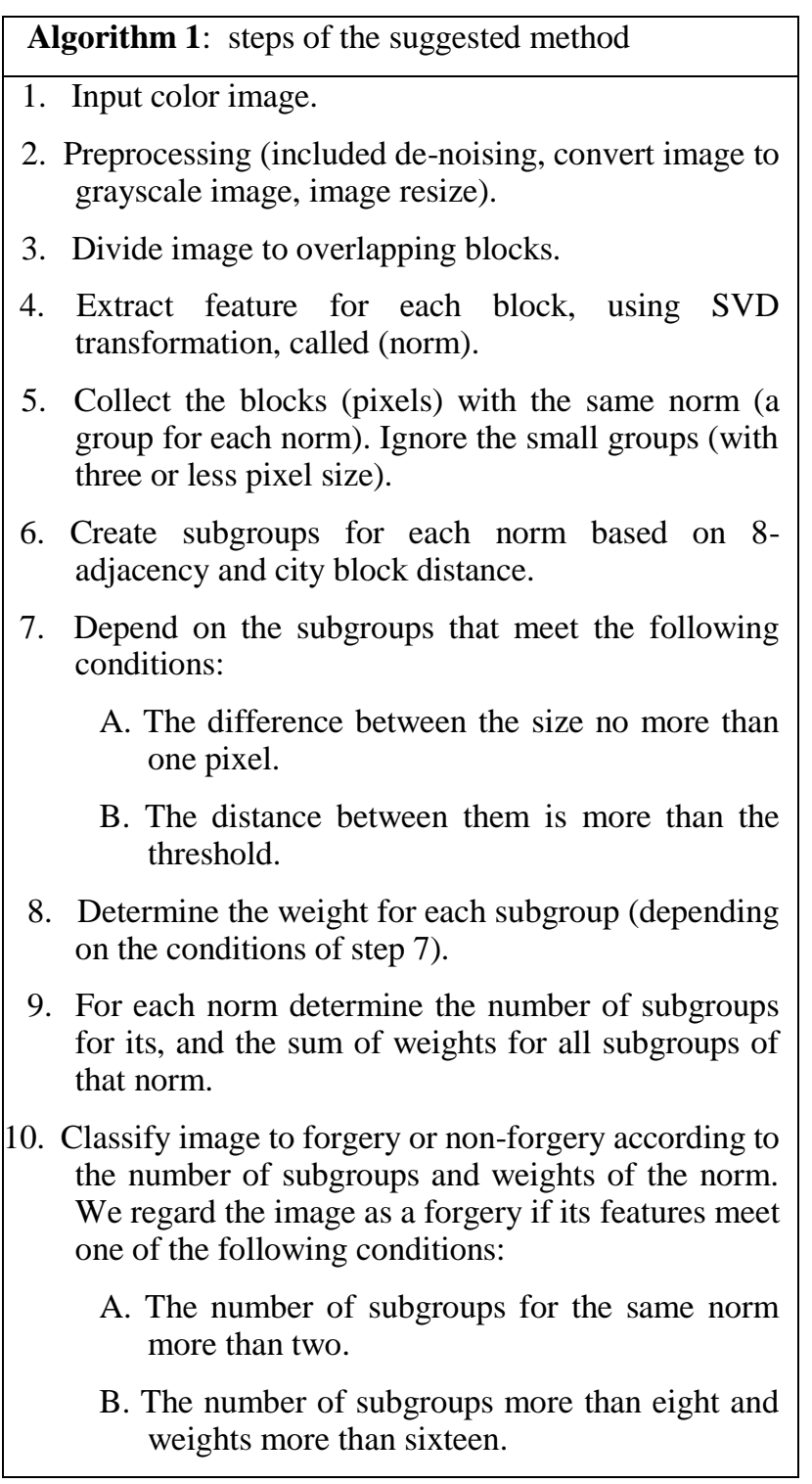

Block diagram of the algorithm 1 shown in Fig. 1.

The first step in this proposal is preprocessing. In this step, the input image is a color image, which is converted to a grayscale image. Then, the de-noising was implemented on the image by using a $3 \times 3$ mean filter. The size of the image will be resized to (256x384) (this is the size of most images in the dataset), while the image with a smaller size than $256 \times 384$ will leave as it is.

The input image will be divided into $16 \times 16$ overlap blocks. For each block the SVD determined, it is obvious in this step we get three matrices from 


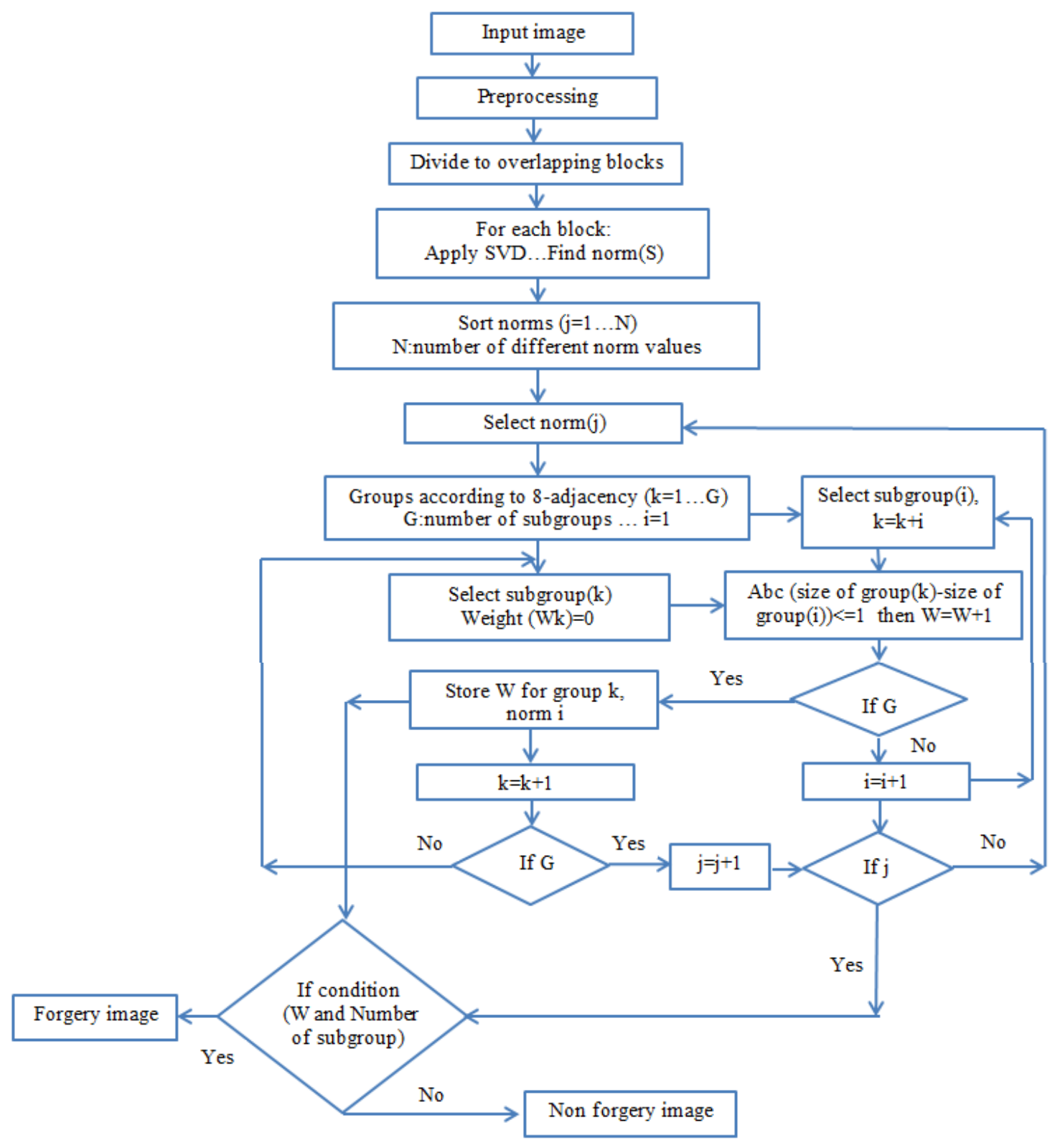

Figure. 1 Block diagram for the proposed algorithm

the SVD process. The norm value (used as matching value) of the diagonal matrix will be determined.

$$
\text { norm }=\sqrt{\sum_{i=1}^{n} S_{i}}
$$

Where

$\mathrm{n}$ : is the number of columns in the diagonal matrix

$\mathrm{S}$ : is the singular value.

Norm values are sorted in ascending order. The pixels with similar norm values will be collected together (number of groups depend on the number of different norm values). Groups with three or fewer members (pixels) will be ignored. Each group is dividing again into subgroups. All the pixels with the same norm and have 8-adjacency will be grouped.
This step aims to find the connected pixels which may represent an object. Ultimately, the subgroups that will be dependent (undergo the forgery detection), should have a distance between them less than or equal to thirty (measured by city block distance).

The subgroups for each norm will be filtered to select just the subgroups which can be nominated as identical and separated regions (copy regionls). These subgroups have more than two pixels; other subgroups will be ignored. Now, each subgroup is compared with all the other subgroups with the same norm. The weight for selected subgroup will be increased by one if it is achieved the following two conditions when compared with another subgroup: 
First: the difference between the size (number of pixels) of the selected subgroup with another subgroup should be no more than one.

Second: the distance between the selected subgroup and the other subgroup more than seventeen.

At the end of comparing all the subgroups with others, each subgroup will possess a weight (the weight of each norm is the sum of weights of all subgroups). Also, the number of nominated subgroups may be decreased, we have to know the number of subgroups remains for each norm.

Two one-dimension arrays will be created, each one included all the norms value, these two arrays sorted in descending order. The first one (W1) sorted according to the weights of the norm. While the second array (W2) sorted according to the number of subgroups left (nominated) in each norm (norm with the highest number of subgroups first). From (W1) we select just the highest six group (the first six norms, and neglect the others), while from the (W2) we select just the first ten of sorted array (highest number of subgroups).

The subgroup (region) regards as forgery region (copy and move) when achieved one of the following conditions:

1. The number of subgroups (in the first ten norms of array W2) more than or equal eight, and the weight (in the first six norms of array W1) is greater than or equal sixteen.

2. The number of subgroups (in the first ten norms of array W2) more than or equal eight and the number of subgroups (in the first six norms of array W2) is less than or equal eight.

Otherwise, classify as non-forgery images.

\section{Results and discussion}

At this work, we enforce many conditions and ideas to achieve high accuracy. These conditions emerged from experiments. In this section, we summarize all the experiments to test the image forgery detection accuracy. The image dataset we work on is Casia.

The first experiment was to check the best image size for the suggested algorithm. The size of images in the dataset almost is $(256 \times 384)$ and there are some images larger and smaller than size. Resizing the larger images to $(256 \times 384)$ will give good results to detect the forgery images, but some of the nonforgery images will not be detected, if we continue to reduce the image size this will lead to detect nonforgery images and degrade of accuracy for detection forgery images, as shown in the Fig. 2. For that, all

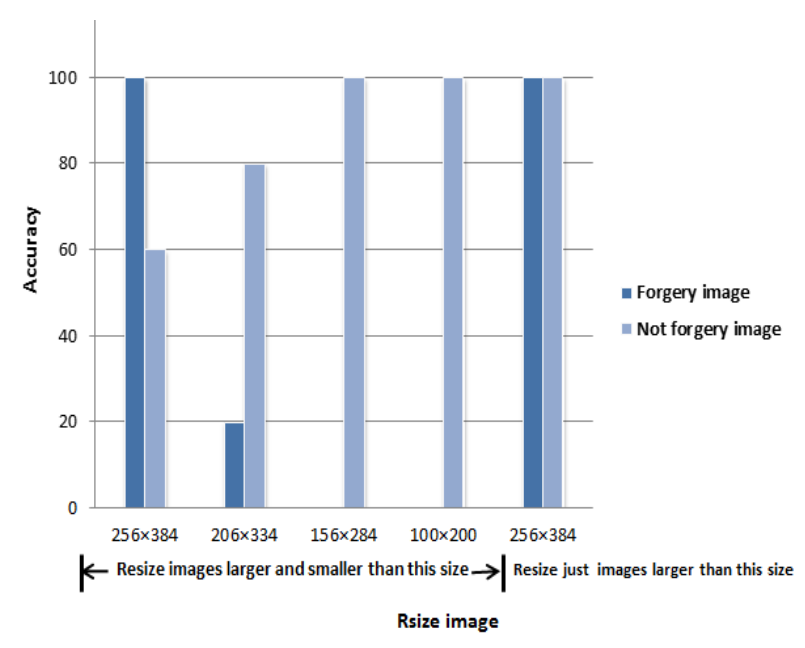

Figure. 2 The relation between resizing image and accuracy

the large images will be resized, while the smallest images have not changed.

The second test was to find the best block size, we check the accuracy for different block sizes as shown in Fig. 3. We conclude that the accuracy will increase with increasing the block size until the size $16 \times 16$, more than $16 \times 16$ will decrease the accuracy for detection forgery and non-forgery images.

Also, we determined how the distance between subgroups effect on the accuracy. We need to be sure that the separated subgroups of the same norm represent the possibility of copy regions. Fig. 4 shows the best distance for the best accuracy.

The separated subgroups for the same norm regard as a sign for forgery regions. So, we test how the number of subgroups effects on forgery detection. This test is implemented on the array of ten norms (W2). From Fig. 5, we conclude that the detection of forgery images is excellent when including the norms with a few numbers of subgroups, the accuracy is well until it only includes a larger number of subgroups (more than sixteen). From the other side, the detection of non-forgery images becomes better when only includes a large number of subgroups. As a result, the best accuracy when the number of subgroups is equal to or more than eight.

Also, we see from Fig. 5 that we get the best accuracy for array (W2) when the number of subgroups equal to or more than eight. In this case, we try to reduce the computations by determining the number of norms included in array W2 to achieve this accuracy.

From Fig. 6 we found that the number of norms equal to ten is quite enough to meet this accuracy.

Array W1 created to include the weights of norms, to see how these array effect on the detection of forgery, we test the weight as a parameter to detect 


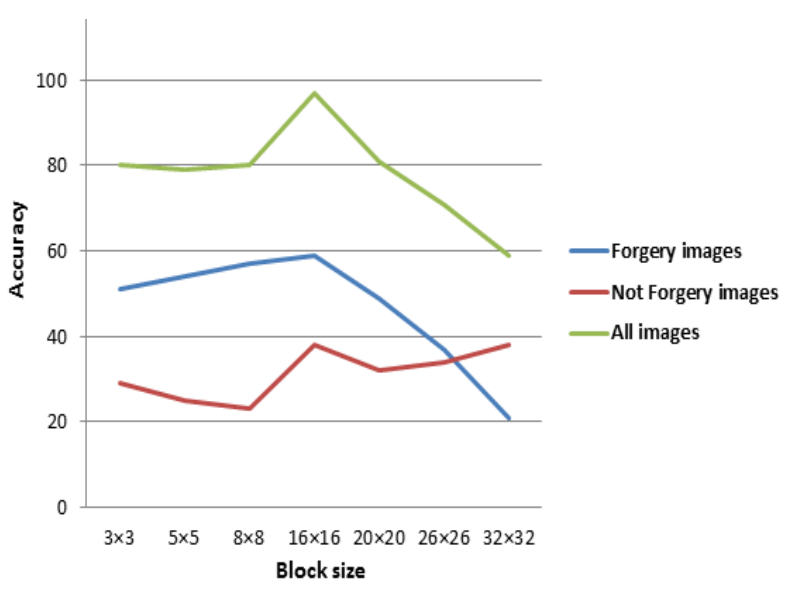

Figure. 3 The relation between block size and accuracy

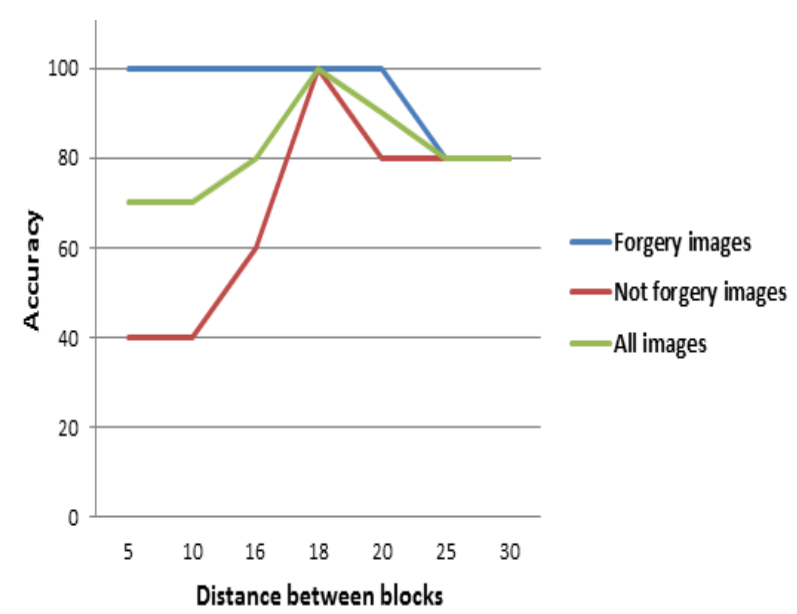

Figure. 4 The relation between accuracy and distance between blocks

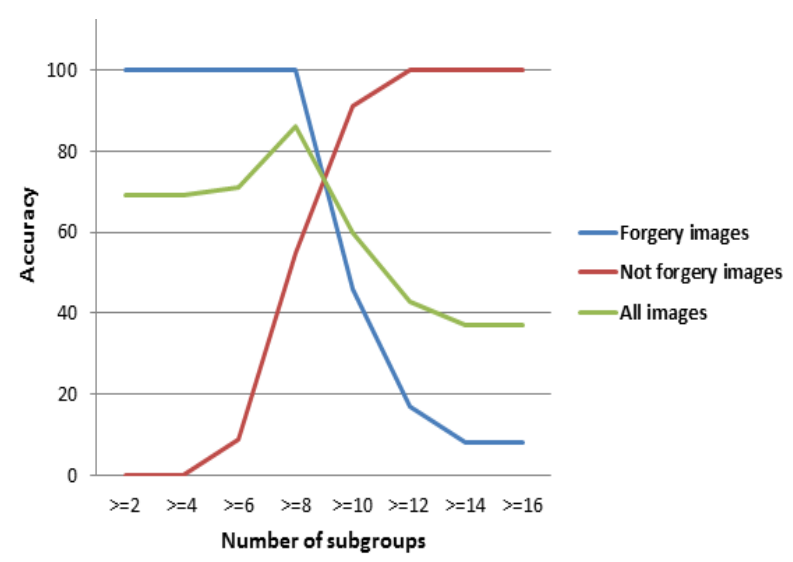

Figure. 5 Effect of the number of subgroups on the forgery detection

the forgery images. Fig. 7 shows the results of this test. We conclude that accuracy varies according to the increasing value of weight. When the array included the small values of the weight, the detection of non-forgery was reduced. With including only, the high weights the detection of non-forgery increased, but the detection of forgery decreased. We tradeoff

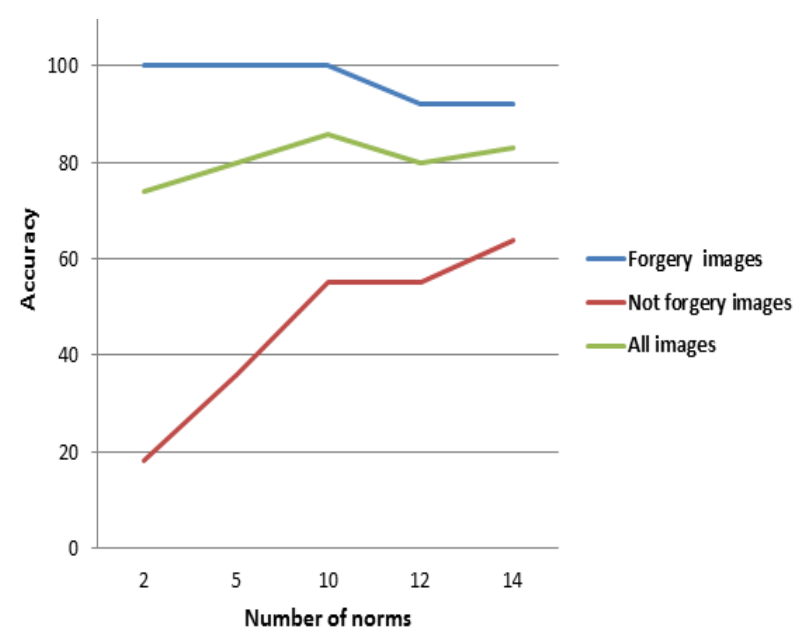

Figure. 6 Number of norms for array W2

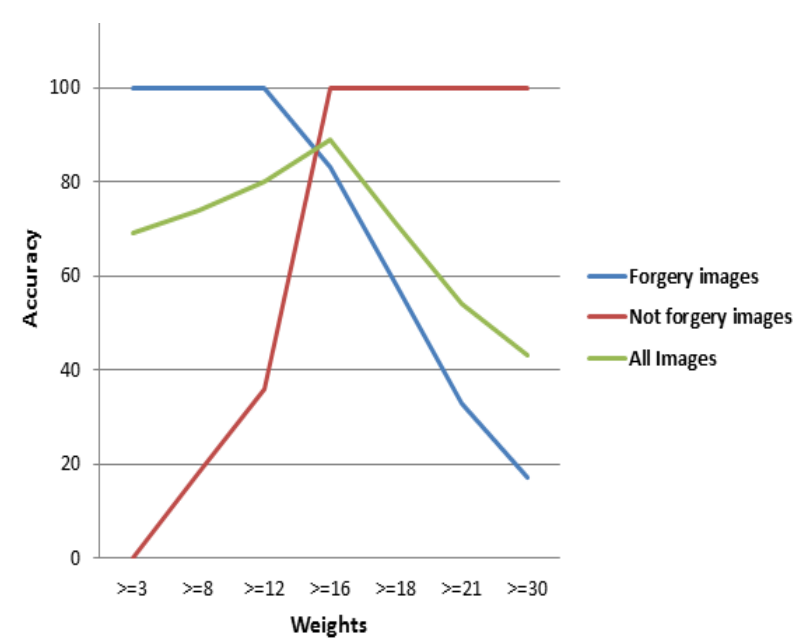

Figure. 7 The weight effect on forgery detection accuracy

between forgery and non-forgery, and regards the weight equal or greater than sixteen is the best.

To reduce computation, we determine the minimum number of norms for array $\mathrm{W} 1$ that can achieve accuracy in Fig. 7. The accuracy-related to the number of norms that can be selected to test forgery images according to the weight shows in Fig. 8. Six norms give the best results for detection and classification forgery and non-forgery images.

As we see from Fig. 5, detection accuracy for non-forgery images is well when the number of subgroups equal or larger than eight, while the detection of forgery images is best when we included the small numbers of subgroups. For that, we try to check the accuracy just for small numbers of subgroups without including the high numbers of subgroups as shown in Fig. 9. We conclude that using several subgroups less than or equal to eight will help to detect non-forgery images. Also, we see that the eight number of subgroups is the separated point between detection only forgery images or detection only non-forgery images. 


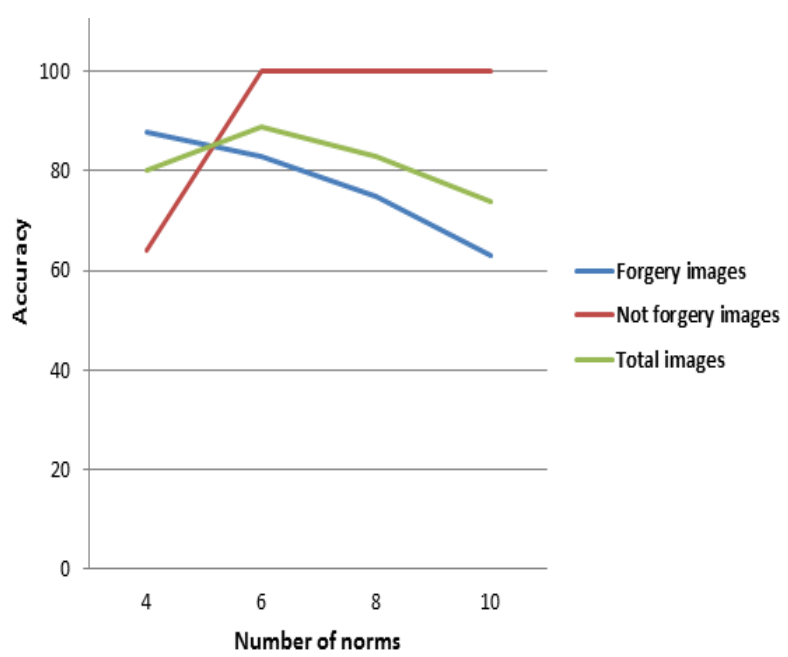

Figure. 8 Determine the minimum number of norms for array $\mathrm{W} 1$

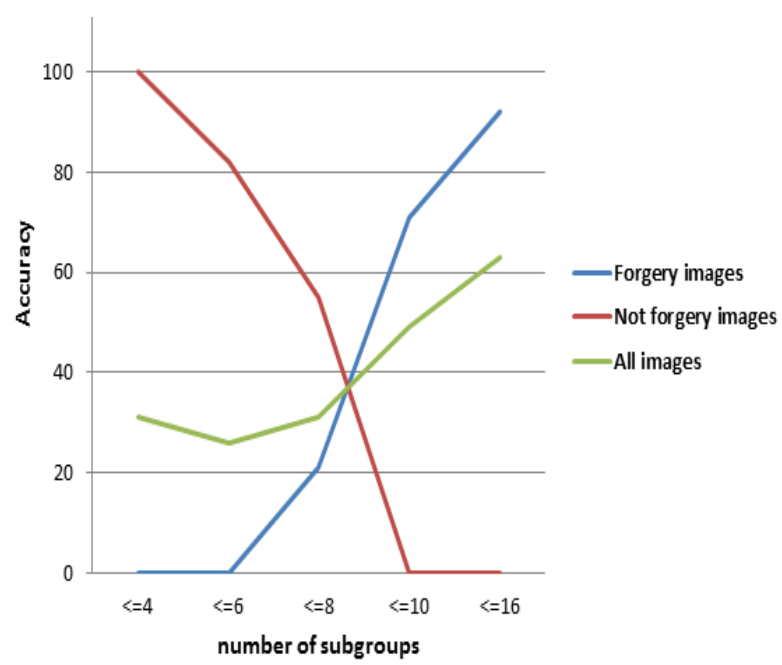

Figure. 9 Detection accuracy when using only a small number of subgroups

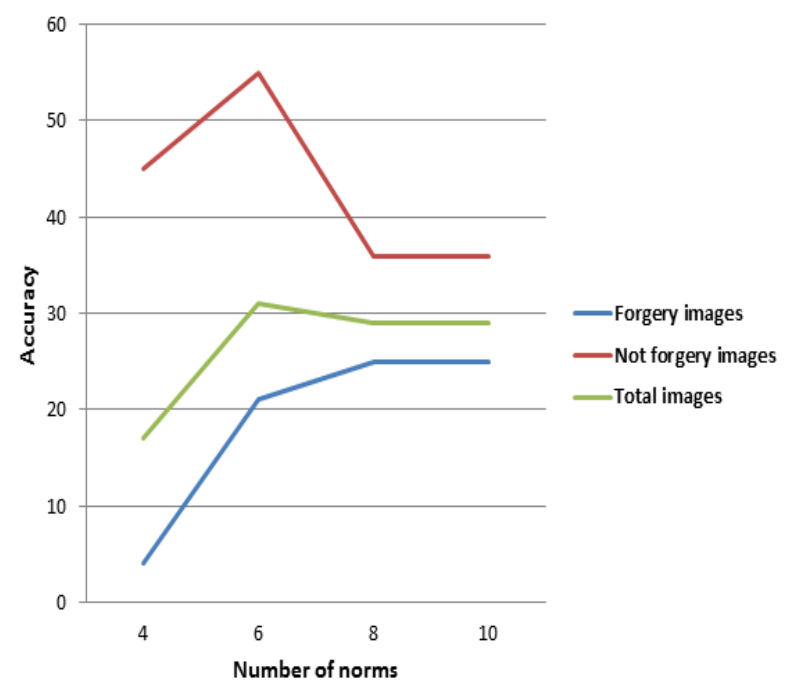

Figure. 10 Number of norms for the number of subgroups less or equal eight

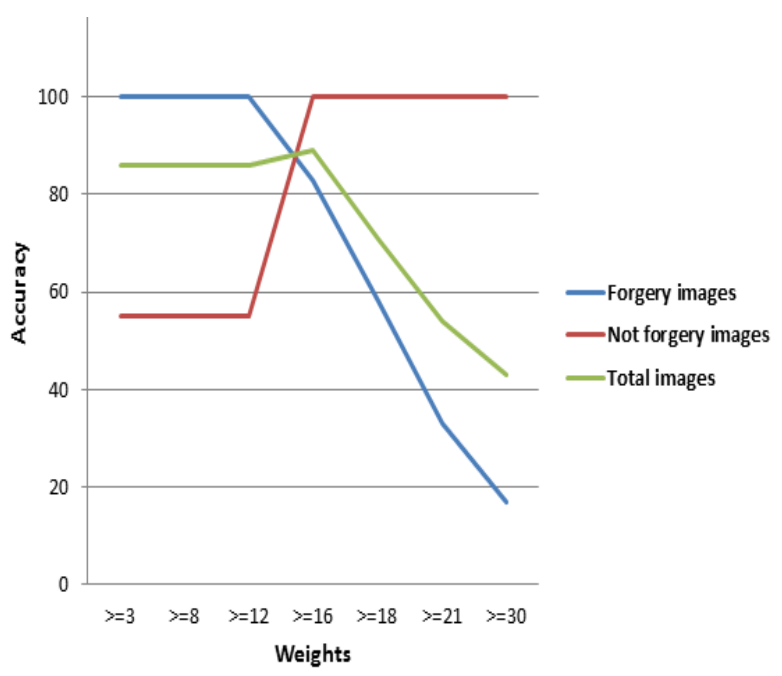

Figure. 11 Test variation of accuracy with different weights when the number of subgroups greater than eight

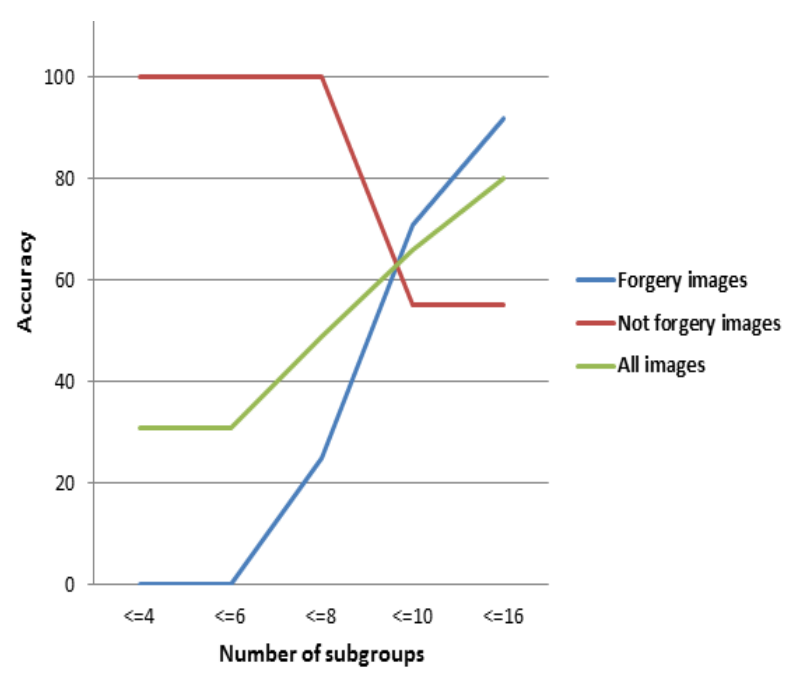

Figure. 12 Accuracy when combining two conditions (norms with subgroups more than eight and norms of a small number of subgroups)

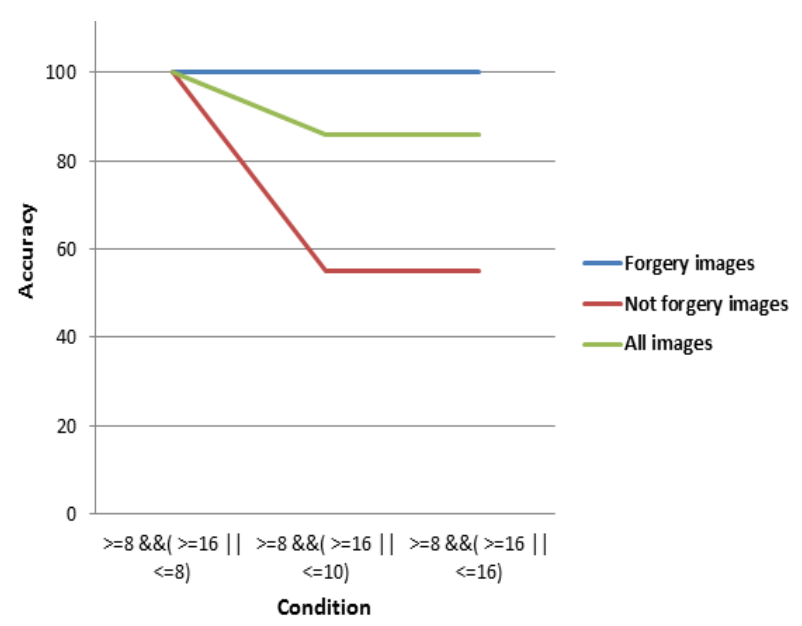

Figure.13 Test accuracy when combining the main two conditions 
The number of norms to achieve the condition in Fig. 9 may be changing, we have to check that with different norms as in Fig. 10. Many norms six or less will give better results.

Another test to check how the accuracy various when we combine two condition (number of subgroups $>=8$, and different weights). Fig. 11 show that the detection of forgery images was very good for weights less than sixteen, opposite to non-forgery which increased when weights more than sixteen, the best result is to choose the weights equal or greater than sixteen.

Now, what happens to accuracy when combining the norms with many subgroups equal to or greater than eight, and norms with only small numbers of subgroups. Fig. 12 shows the result of this test.

As a final test to decide the best conditions for detection forgery image, we combine the main two conditions, the first one is to include all the subgroups or combine the number of subgroups equal or greater than eight and the weights more than sixteen. as shown in Fig. 13.

The final accuracy for detection and classification forgery and non-forgery images is 97\%. Fig. 14 shows a sample of detected forgery images.
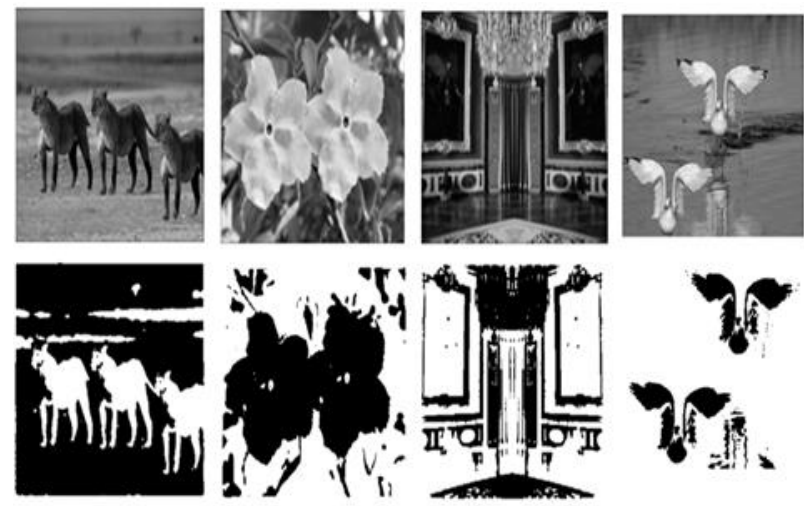

Figure. 14 Sample of detected forgery images
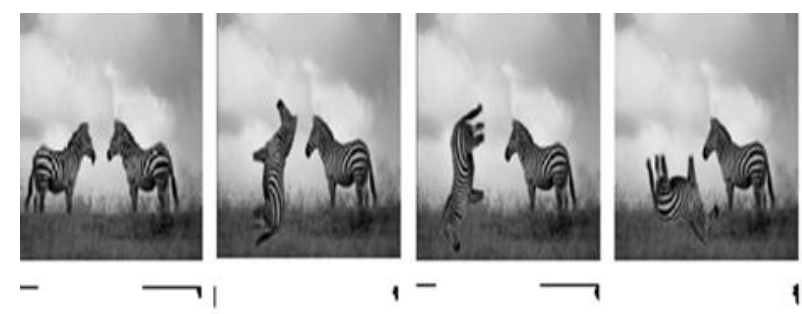

$1-$
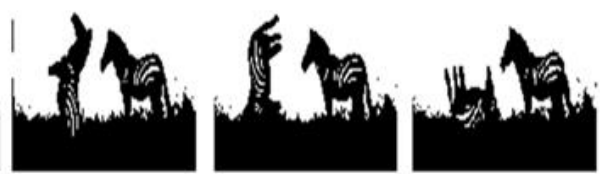

Figure. 15 Sample of detected forgery images when rotating forgery objects with different angles
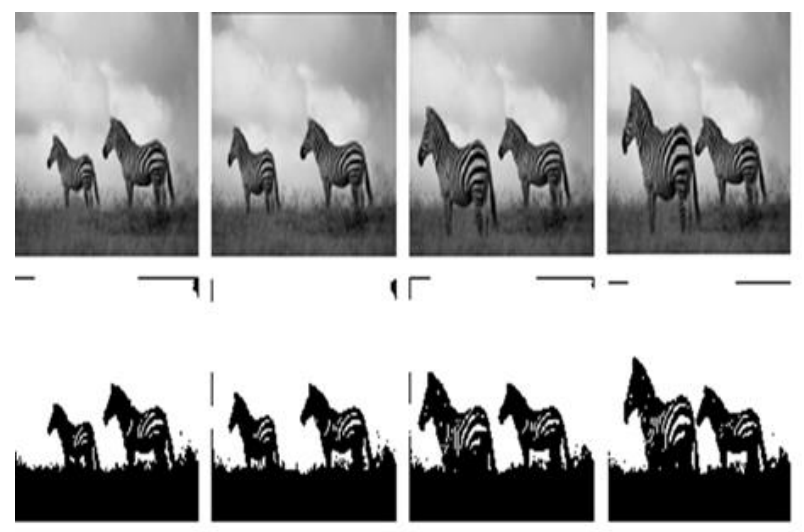

Figure. 16 Sample of detected forgery images when scaled the object
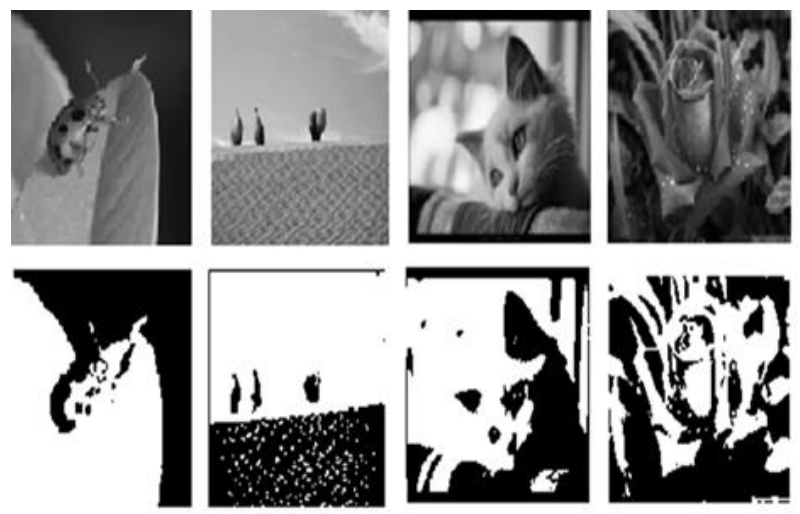

Figure. 17 Sample of non-forgery images detected by the suggested method
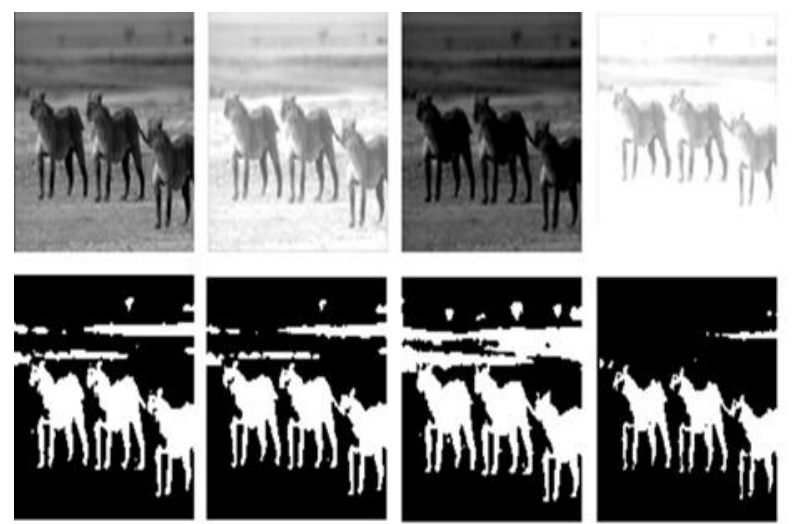

Figure. 18 Sample of detected forgery image with different illumination

Also, we checked the robustness of the suggested algorithm against rotation and scaling with different degrees. The suggested algorithm is highly robust for rotation and scaling and successes all the images that rotate the tempered object or scale it. Fig. 15 show sample of detection forgery images with rotation objects with different angles, and Fig. 16 show sample of detected forgery images when scaling forgery object. Where Fig. 17 show a sample of nonforgery images, which all detected successfully. Scaling and rotation for any block have no direct 
effect of the value of norm when using the SVD as a matching value.

The suggested method tested the accuracy to detect forgery images when the variation of image illumination, changing of illumination is a big problem used to conceal the forgery, and most of the other methods did not solve the variation of illumination.

Table 1. Comparing the proposed method with other similar methods

\begin{tabular}{|c|c|c|c|c|c|c|c|c|}
\hline Author(s) & Technique & Dataset & $\frac{0}{e_{0}^{0}}$ & 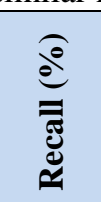 & $\begin{array}{c}\mathrm{F} \\
(\%)\end{array}$ & 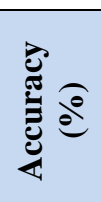 & 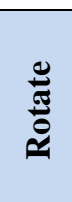 & 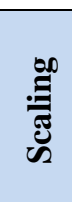 \\
\hline $\begin{array}{c}\text { Kashya, Agarwal, } \\
\text { and Gupta, (2018) } \\
\text { [9] }\end{array}$ & $\begin{array}{l}\text { Singular Value } \\
\text { Decomposition } \\
\text { (SVD) and Cuckoo } \\
\text { Search Algorithm }\end{array}$ & $\begin{array}{c}\text { CoMoFoD } \\
\text { database (DB- } \\
\text { 1), MICC-F600 } \\
\text { (DB-2), MICC- } \\
\text { F2000 (DB-3) }\end{array}$ & 96.13 & 92.3 & 94.18 & & No & Yes \\
\hline $\begin{array}{c}\text { Bi and Pun, (2018) } \\
{[6]}\end{array}$ & $\begin{array}{l}\text { Local bidirectional } \\
\text { coherency error }\end{array}$ & $\begin{array}{c}\text { CMFD_A and } \\
\text { CMFD_B } \\
\text { datasets }\end{array}$ & & & 96.63 & & Yes & Yes \\
\hline $\begin{array}{l}\text { Isaac and Wilscy, } \\
\text { (2018) [8] }\end{array}$ & $\begin{array}{l}\text { edge-based features, } \\
\text { Saliency, Wavelets, } \\
\text { and Rotation invariant } \\
\text { Co-occurrences } \\
\text { among adjacent LBP } \\
\text { (RiCLBP) }\end{array}$ & $\begin{array}{l}\text { CASIA v1.0, } \\
\text { CASIA v2.0, } \\
\text { and CUISDE } \\
\text { datasets }\end{array}$ & & & & 97.15 & No & No \\
\hline $\begin{array}{c}\text { Bi and Pun, (2017) } \\
\text { [13] }\end{array}$ & $\begin{array}{l}\text { the reflective offset- } \\
\text { guided searching }\end{array}$ & $\begin{array}{c}\text { CMFD_A and } \\
\text { CMFD_B } \\
\text { datasets }\end{array}$ & & & 96.63 & & Yes & Yes \\
\hline $\begin{array}{l}\text { Bi, Pun, and Yuan, } \\
\text { (2016) [14] }\end{array}$ & $\begin{array}{l}\text { Multi-Level Dense } \\
\text { Descriptor (MLDD) } \\
\text { and Hierarchical } \\
\text { Feature Matching }\end{array}$ & CMFDA dataset & 88.89 & 100 & 94.12 & & Yes & Yes \\
\hline $\begin{array}{c}\text { Malviya and } \\
\text { Ladhake ,(2016)[15] }\end{array}$ & $\begin{array}{c}\text { Auto Color } \\
\text { Correlogram (ACC) }\end{array}$ & $\begin{array}{l}\text { CoMoFoD } \\
\text { database }\end{array}$ & 95.65 & 91.67 & 93.62 & & Yes & Yes \\
\hline $\begin{array}{c}\text { Dubey, Sarawagi, } \\
\text { and Shrivastava, } \\
\text { (2016) [16] }\end{array}$ & $\begin{array}{l}\text { wavelet transform } \\
\text { function and } \\
\text { Clustering technique }\end{array}$ & UCID dataset & & & & 97.43 & Yes & Yes \\
\hline $\begin{array}{c}\text { Oommen, } \\
\text { Jayamohan, and } \\
\text { Sruthy, (2016) [17] }\end{array}$ & $\begin{array}{c}\text { Local Fractal } \\
\text { Dimension; B+ tree; } \\
\text { Singular Value } \\
\text { Decomposition }\end{array}$ & Private dataset & 91.39 & 95.5 & & & Yes & No \\
\hline $\begin{array}{l}\text { Alahmadi, Hussain, } \\
\text { Aboalsamh, } \\
\text { Muhammad, Bebis, } \\
\text { and Mathkour, } \\
\text { (2016) [18] }\end{array}$ & $\begin{array}{l}\text { local binary pattern } \\
\text { (LBP), discrete cosine } \\
\text { transform (DCT) and } \\
\text { support vector } \\
\text { machine(SVM) }\end{array}$ & $\begin{array}{l}\text { CASIA TIDE } \\
\text { v1.0, CASIA } \\
\text { TIDE v2.0, and } \\
\text { Columbia }\end{array}$ & & & & 97.00 & Yes & No \\
\hline $\begin{array}{l}\text { Hashmi , Anand, } \\
\text { and } \\
\text { Keskarc ,(2014)[19] }\end{array}$ & $\begin{array}{c}\text { Dyadic Wavelet } \\
\text { Transform (DyWT), } \\
\text { Scale Invariant } \\
\text { Feature Transform } \\
\text { (SIFT) }\end{array}$ & $\begin{array}{l}\text { MICC-F220 } \\
\text { dataset }\end{array}$ & 88 & 80 & & & Yes & Yes \\
\hline $\begin{array}{c}\text { Muhammad, Al- } \\
\text { Hammadi, Hussain, } \\
\text { and Bebis, (2014) } \\
{[20]}\end{array}$ & $\begin{array}{l}\text { Steerable pyramid } \\
\text { transform, Local } \\
\text { binary pattern }\end{array}$ & $\begin{array}{l}\text { CASIA v1.0, } \\
\text { CASIA v2.0, } \\
\text { and Columbia } \\
\text { color DVMM }\end{array}$ & 97.87 & 86.78 & & 93.80 & Yes & Yes \\
\hline Proposed method & $\begin{array}{c}\text { Singular Value } \\
\text { Decomposition } \\
\text { (SVD) }\end{array}$ & $\begin{array}{l}\text { CASIA v1.0 } \\
\text { and CASIA } \\
\text { v2.0 datasets }\end{array}$ & 97 & 98 & 97.49 & 97 & Yes & Yes \\
\hline
\end{tabular}


The suggested algorithm successes to detect all the forgery images despite different illumination, Fig. 18 show a sample of detected forgery images with different illumination. This is one of the strong points of using the SVD as a matching value, where the change in the illumination of the image has the same effect on all pixels, and that will lead to the samechange on the value of the norm.

The suggested method is compared with other methods. Table 1 summarizes the accuracy and important points for different methods. The suggested method has promised accuracy.

\section{Conclusion}

Detection of the copy-move forgery images by using SVD is proposed in the current research. The current method has high accuracy to detect forgery images and discriminated from non-forgery images. The contributions of this paper are the robustness for detection forgery when rotating the tempered object(s) with any angle, also detect forgery when scaling object(s) with different sizes (shrink or enlarge). The suggested method can detect more than one tempered object in a single image. In this method change of illumination did not affect the accuracy for the detection of the forgery images.

Image forgery detection by using SVD was very good comparing with previous works.

The performance of the suggested algorithm was promised according to the performance measures (Precision (97\%), Recall (98\%), F1 (97.49\%), Accuracy (97\%)).

The average time required to detect forgery images is about $32 \mathrm{sec}$. and can be reduced when using high efficient computers.

Using the SVD as a matching value will enhance the process of matching blocks because each pixel will be matched with other pixels according to the value of norm which is related directly to the value of all pixels in the block, so any uniform change of pixels value does not affect the value of norm.

For the future work, detecting other types of forgery such as Retouching and Splicing forgery by using the same technique. Use other techniques with SVD techniques such as local binary patterns may increase accuracy.

\section{Conflicts of Interest}

The authors declare no conflict of interest.

\section{Author Contributions}

The authors' contributions are as follows: "conceptualization, Nidhal K. El Abbadi, and Alyaa
Mohsin; methodology, Nidhal K. El Abbadi; software, Alyaa Mohsin; validation, Nidhal K. El Abbadi, and Alyaa Mohsin; formal analysis, Alyaa Mohsin; investigation, Nidhal K. El Abbadi; resources, Alyaa Mohsin; data curation, Nidhal K. El Abbadi, and Alyaa Mohsin; writing - original draft preparation, Alyaa Mohsin; writing-review and editing, Nidhal K. El Abbadi; visualization, Nidhal K. El Abbadi; supervision, Nidhal K. El Abbadi; project administration, Nidhal K. El Abbadi; funding acquisition, Alyaa Mohsin”.

\section{References}

[1] S. B. Basavarajappa and S. V. Sathyanarayana, "Digital image forgery detection techniques: a survey", Accents Transactions on Information Security, Vol. 2, pp. 22-31,2017.

[2] J. A. Yadav and N. Dongre, "Analysis of CopyMove Forgery Detection in Digital Image", International Journal of Engineering Development and Research, Vol. 5, No. 1, pp. 732-736, 2017.

[3] Mehak and T. Gulati, "Detection of Digital Forgery Image using Different Techniques", International Journal of Engineering Trends and Technology (IJETT), Vol. 46,No. 8, pp. 457-461, 2017.

[4] M. N. O. Sadiku, S. M. Musa, and S. R. Nelatury, "Digital Forgery", International Journal of Advances in Scientific Research and Engineering (ijasre), Vol. 3, No. 4, pp. 26-29, 2017.

[5] S. Sadeghi, S. Dadkhah, H. A. Jalab, G. Mazzola, and D. Uliyan, "State of the art in passive digital image forgery detection: copy-move image forgery", Pattern Analysis and Applications, Vol. 21, pp. 291-306, 2017.

[6] X. Bi and C. M. Pun, "Fast Copy-Move Forgery Detection Using Local Bidirectional Coherency Error Refinement", Pattern Recognition, Vol. 81, pp. 161-175, 2018.

[7] C. M. Pun and J. L. Chung, "A two-stage localization for copy-move forgery detection", Information Sciences, Vol. 463, pp. 33-55, 2018.

[8] M. M. Isaac and M. Wilscy, "Image forgery detection using region-based Rotation Invariant Co-occurrences among adjacent LBPs", Journal of Intelligent \& Fuzzy Systems, Vol. 34, pp. 1679-1690, 2018.

[9] A. Kashyap, M. Agarwal, and H. Gupta, "Detection of copy-move image forgery using SVD and cuckoo search algorithm", International Journal of Engineering \& Technology, Vol. 7, pp. 79-87, 2018. 
[10] F. Liu and H. Feng, "An efficient algorithm for image copy-move forgery detection based on DWT and SVD", International Journal of Security and Its Applications, Vol. 8, No. 5, pp. 377-390, 2014.

[11] K. Asghar, Z. Habib, and M. Hussain, "Copymove and splicing image forgery detection and localization techniques: a review", Australian Journal of Forensic Sciences, Vol. 49, No. 3, pp. 281-307, 2017.

[12] N. K. El Abbadi, A. Mohamad, and M. AbdulHameed, "Image Encryption Based On Singular Value Decomposition", Journal of Computer Science, Vol. 10, No. 7, pp. 1222-1230, 2014.

[13] X. Bi and C. M. Pun, "Fast Reflective OffsetGuided Searching Method for Copy-Move Forgery Detection", Information Sciences, Vol. 418-419, pp. 531-545, 2017.

[14] X. Bi, C. M. Pun, and X. C. Yuan, "Multi-level dense descriptor and hierarchical feature matching for copy-move forgery detection", Information Sciences, Vol. 345, pp. 226-242, 2016.

[15] A. V. Malviya and S. A. Ladhake, "Pixel-based Image Forensic Technique for copy-move forgery detection using Auto Color Correlogram", In: Proc. of International Conf. on Communication, Computing and Virtualization (ICCCV) 2016, Vol. 79, pp. 383390, 2016.

[16] S. Dubey, A. Sarawagi, and M. Shrivastava, "Image Forgery Detection Based on Local Descriptors and Block-Matching using Clustering Technique", International Journal of Computer Applications, Vol. 141. No, 10, pp. 11-14, 2016.

[17] R. S. Oommen, M. Jayamohan, and S. Sruthy, "Using Fractal Dimension and Singular Values for Image Forgery Detection and Localization", In: Proc. of International Conf. on Emerging Trends in Engineering, Science and Technology (ICETEST), Trissur, India, Vol. 24, pp. 1452$1459,2016$.

[18] A. Al-Ahmadi, M. Hussain, H. Aboalsamh, G. Muhammad, G. Bebis, and H. Mathkour, "Passive detection of image forgery using DCT and local binary pattern", Signal, Image and Video Processing, Vol. 11, pp. 81-88, 2016.

[19] M. F. Hashmi, V. Anand, and A. G. Keskarc, "Copy-move Image Forgery Detection Using an Efficient and Robust Method Combining UnDecimated Wavelet Transform and Scale Invariant Feature Transform", In: Proc. of AASRI Conf. on Circuit and Signal Processing (CSP), Vol. 9, pp. 84-91, 2014.
[20] G. Muhammad, M. H. Al-Hammadi, M. Hussain, and G. Bebis, "Image forgery detection using steerable pyramid transform and local binary pattern", Machine Vision and Applications, Vol. 25, No. 4, pp. 985-995, 2014. 